\title{
Avaliação do impacto do turismo sobre ambiente recifal costeiro inserido em área marinha protegida
}

\section{Leandro do Nascimento Máximo ${ }^{1}$, Daniel Silva Lula Leite ${ }^{2}$ e George Emmanuel Cavalcanti de Miranda ${ }^{3}$}

\author{
${ }^{1}$ Programa de Pós-Graduação em Ecologia e Monitoramento Ambiental. \\ Universidade Federal da Paraíba. Rio Tinto-PB, Brasil (CEP 58297-000). E-mail: \\ leomaximo147@gmail.com. \\ ${ }^{2}$ Programa de Pós-Graduação em Desenvolvimento e Meio Ambiente. Universidade \\ Federal da Paraíba. João Pessoa-PB, Brasil (CEP 58051-900). \\ ${ }^{3}$ Departamento de Sistemática e Ecologia. Centro de Ciências Exatas e da Natureza. \\ Universidade Federal da Paraíba. João Pessoa-PB, Brasil (CEP 58051-900).
}

Resumo. Os ambientes recifais costeiros têm sofrido com a expansão da atividade turística sobre esta zona. 0 objetivo deste trabalho é avaliar o impacto do turismo sobre a estrutura da comunidade de macroalgas do recife de Picãozinho, Paraíba, Brasil, anteriormente à sua inserção na Área de Proteção Ambiental (APA) Naufrágio Queimado. Foram realizadas coletas durante os períodos seco e chuvoso nos recifes de Picãozinho (com visitação turística) e de São Gonçalo (sem visitação turística), avaliando a riqueza, diversidade, dominância e biomassa total da comunidade. Foram encontrados os maiores valores de riqueza, diversidade e biomassa total em São Gonçalo, com a dominância sendo maior em Picãozinho. Houve maior desenvolvimento do talo em São Gonçalo, com algumas espécies apresentando-se na forma de turf em Picãozinho. 0 turismo pode impactar a estrutura, o funcionamento, a biodiversidade e a resiliência da comunidade recifal, afetando, consequentemente, a qualidade ambiental dos recifes. Fornece-se à gestão da APA Naufrágio Queimado um panorama do impacto da atividade turística praticada no local, objetivando o turismo marinho sustentável.

Palavras-chave: Macroalgas; Ambiente recifal costeiro; Impacto do turismo; Área marinha protegida.

Abstract. Impact assessment of tourism on coastal reef environment in a marine protected area. Reef environments have suffered with the increase of tourist activity in this region. The objective of this work is to evaluate the impact of tourism on the structure of the recovery macroalgal community of Picãozinho, Paraíba, Brazil, prior to its insertion in the Naufrágio Queimado Environmental Protection Area. Samples were collected during the dry and rainy periods in the reefs of
Recebido

23/09/2019

Aceito

23/12/2019

Publicado

$31 / 12 / 2019$

Acesso aberto

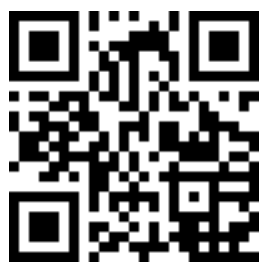

ORCID

D 0000-0003-1597-4505

Leandro do

Nascimento Máximo

D 0000-0001-9104-7418

Daniel Silva Lula Leite

(D) 0000-0002-8975-1067

George Emmanuel

Cavalcanti de Miranda 
Picãozinho (with tourist visitation) and São Gonçalo (without tourist visitation), evaluating community's richness, diversity, dominance and total biomass. The highest values of richness, diversity and total biomass were found in São Gonçalo, with the dominance in Picãozinho. There was a further development of the canopy in São Gonçalo, with some turf shaped species in Picãozinho. Tourism can impact on the structure, functioning, biodiversity and resilience of the reef community, thereby affecting the environmental quality of the reefs. Provide to the Naufrágio Queimado Environmental Protection Area management an overview of the impact of on-site tourism activity, targeting sustainable marine tourism.

Keywords: Macroalgae; Coastal reef environment; Tourism impact; Marine protected area.

\section{Introdução}

Ecossistemas costeiros são susceptíveis a impactos antropogênicos devido à grande concentração populacional e às diversas atividades realizadas nessa região, como atividades portuárias, pesca, turismo, além da poluição (Ortega, 2000; Leão et al., 2003; Costa et al., 2007). Dentre os principais ecossistemas costeiros e possuindo elevada fragilidade (Graham et al., 2006), os ambientes recifais são prioridades em planos de conservação marinha, sendo comum a criação de áreas protegidas em locais que possuem recifes (Jennings, 2001).

No Brasil, os recifes ocorrem por cerca de $3.000 \mathrm{~km}$ ao longo da costa (Ferreira e Maida, 2006). Na Paraíba, os ambientes recifais distribuem-se ao longo de grande parte da costa, sendo atrações turísticas importantes no estado (Costa et al., 2007). No entanto, os impactos decorrentes das atividades turísticas como pisoteio, movimentação e ancoragem de embarcações, lixo, dentre outros (Lourenço, 2010); aliados aos efeitos sinérgicos da industrialização, desmatamento (Silva, 2013), pesca artesanal, coletas de algas (Souza et al., 2007), poluição e mudanças climática globais (Castro e Zilberberg, 2016), vêm impactando negativamente os recifes do estado.
A qualidade ambiental de um ecossistema aquático é afetada quando a sua capacidade de absorver o estresse é excedida. Qualquer estresse (físico, químico ou biológico) pode gerar alterações desde o nível fisiológico/metabólico dos indivíduos até as estrutura das comunidades. A presença, ausência ou abundância de espécies podem refletir as características do ambiente onde estão inseridas (Areces Mallea et al., 2015). Em comunidades, descritores como riqueza, diversidade, dominância, equitatividade, dentre outros, têm seu padrão local alterados (Vanclay, 2004; Wells, 2007). A contínua ameaça à qualidade ambiental dos ecossistemas aquáticos pode ser avaliada pelo monitoramento biológico utilizando populações ou comunidades biológicas como ferramentas de diagnóstico: os bioindicadores (Dokulil, 2003, Ar Gall, et al., 2016).

Por serem organismos sésseis, possuírem ampla distribuição e responderem a estressores ambientais através da alteração da dominância e/ou desaparecimento de espécies sensíveis, as macroalgas tornam-se eficientes bioindicadores para a determinação de um status de qualidade ecológica (Dokulil, 2003; Stevenson e Smol, 2003; Pinedo et al., 2007). Fatores como tempestades, poluição, erosão e mudanças na temperatura da água mudam drasticamente a estrutura das comunidades associadas neste ecossistema (McClanahan, 2000; 
Dinsdale e Harriott, 2004; McManus e Polsenberg, 2004). Estudos em comunidades bênticas mostram grande potencial das algas em evidenciar efeitos cumulativos de distúrbios nos ecossistemas marinhos (Pinedo et al., 2007; Akcali e Kucuksezgin, 2011; Azevedo et al., 2011; Vasconcelos et al., 2019).

Neste contexto, o objetivo deste trabalho é avaliar a variação espaçotemporal da comunidade de macroalgas em dois recifes costeiros da Paraíba, Brasil, submetidos a diferentes níveis de atividade turística, sob a hipótese que o turismo impacta negativamente a comunidade de macroalgas. Adicionalmente pretende-se contribuir com a gestão da Área de Proteção Ambiental (APA) Naufrágio Queimado, fornecendo um panorama do impacto da atividade turística praticada no local, objetivando o turismo marinho sustentável.

\section{Materiais e método}

\section{Área de estudo}

Os ambientes recifais de

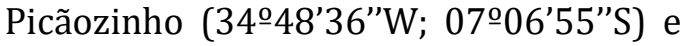
São Gonçalo (34ํ48'48"W; 7ํ06'42"S) (Figura 1) estão localizado no município de João Pessoa (PB), a aproximadamente 1,5 km da Praia de Tambaú e distantes entre si aproximadamente $460 \mathrm{~m}$. Ambos possuem substratos coralíneos que descobrem com a maré abaixo do nível de $0,6 \mathrm{~m}$, formando piscinas naturais (Debeus e Crispim, 2008) de fundo arenoso ou composto por nódulos e partes de corais.

0 recife de Picãozinho recebe aproximadamente 240 turistas diariamente sem nenhum planejamento há mais de 20 anos (Debeus, 2008). Em contrapartida, o recife de São Gonçalo recebe visitação esporádica, represen- tando uma área controle para a atividade turística na Paraíba.

Desde 28 de Dezembro de 2018, os recifes de Picãozinho e de São Gonçalo passaram a ser protegidos pela Área de Proteção Ambiental (APA) Naufrágio Queimado, porém durante a realização do presente estudo a Unidade de Conservação não existia. Os recifes são administrados pela Superintendência de Administração do Meio Ambiente (SUDEMA)em articulação com os órgãos federais, estaduais e municipais, bem como organizações não governamentais (Sudema, 2019).

\section{Estações de coleta e delineamento amostral \\ Foram demarcadas na área de} estudo 5 estações de coleta na região infralitoral raso (aproximadamente $1 \mathrm{~m}$ ) dos recifes(Figura 1): Picãozinho, com as estações P1, P2 e P3 e São Gonçalo com as estações G1 e G2. A seleção contemplou áreas frequentemente visitada por turistas (P1 e P2), área com pouca atividade turística (P3) e áreas sem nenhuma atividade turística, com eventual visita de pescadores (G1 e G2).

As coletas foram realizadas em maio/2014 e outubro/2014 durante a maré baixa de sizígia. Em cada estação de amostragem foram plotados 10 elementos amostrais quadrados de $0,04 \mathrm{~m}^{2}(20 \mathrm{x}$ $20 \mathrm{~cm}$ ), que foram distribuídos aleatoriamente em uma área circular de raio de $20 \mathrm{~m}$. Cada elemento amostral foi plotado a partir do sorteio de um ângulo em graus $\left(15 / 15^{\circ}\right.$, com ângulo $0^{\circ}$ apontado para Norte), e uma distância em metros $0-10 \mathrm{~m}$, com passo de $1 \mathrm{~m}$, a partir do ponto central da circunferência (Figura 2). Quando o elemento amostral se posicionou sobre áreas do médio litoral ou fundo arenoso, foi sorteado outro ponto. 


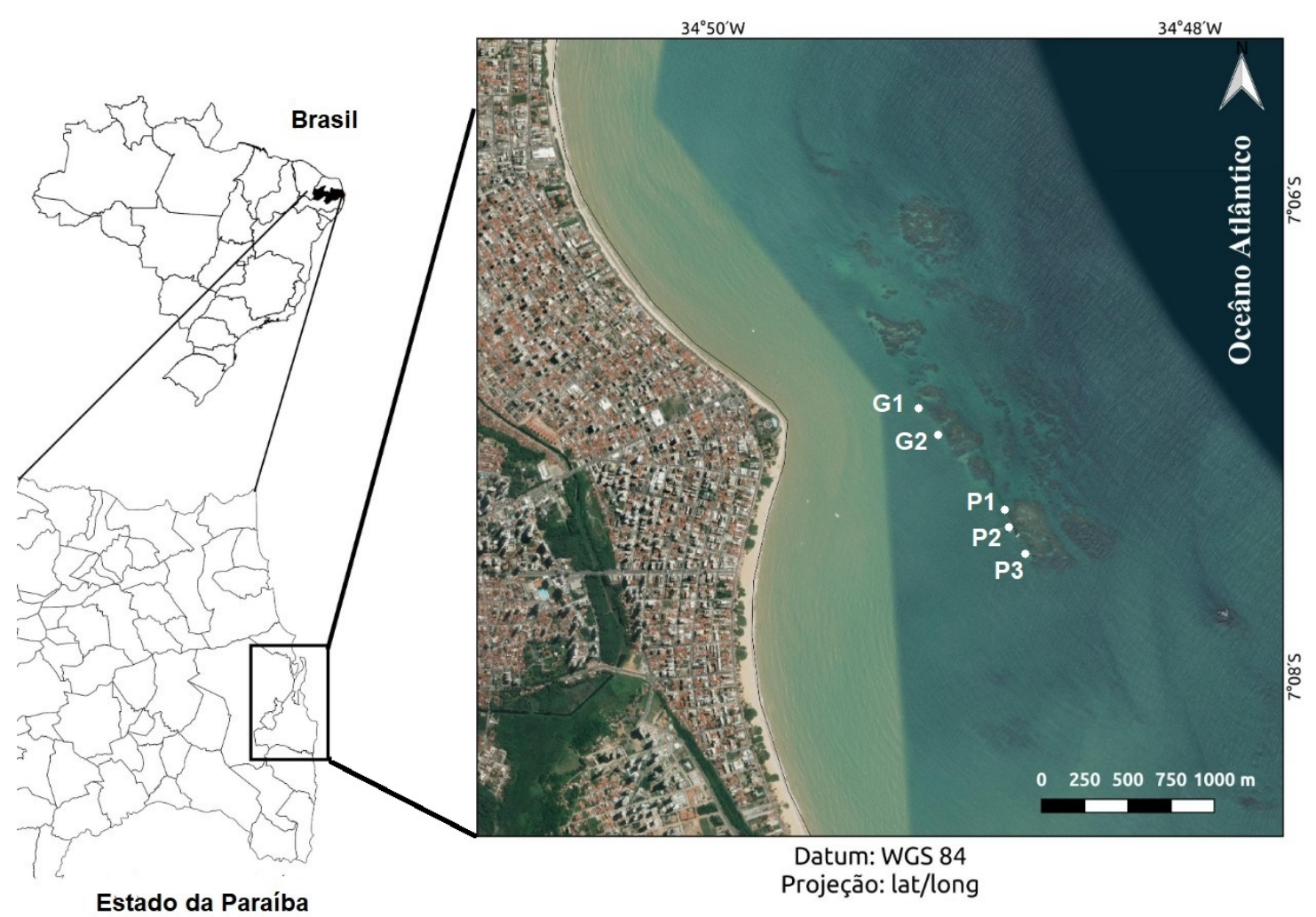

Figura 1. Ambientes recifais objetos de estudo em João Pessoa/PB. Os pontos indicam as estações de coleta em Picãozinho (P1, P2 e P3) e São Gonçalo (G1 e.G2).

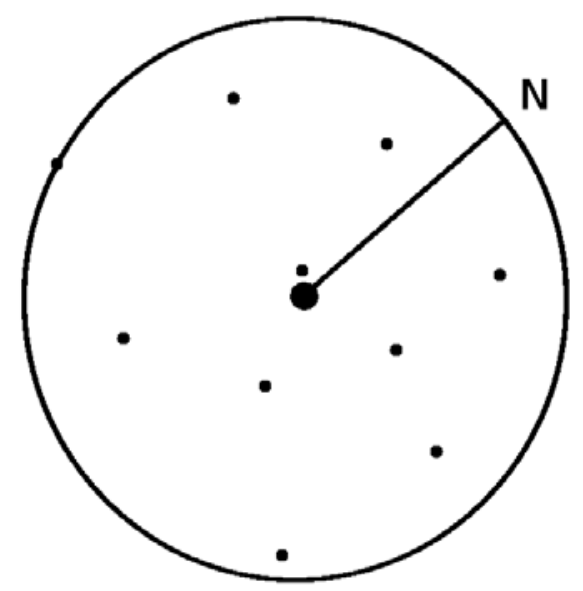

Figura 2. Esquema do desenho amostral utilizado para as coletas em cada estação nas áreas de Picãozinho e São Gonçalo.

Todo o macrofitobento encontrado no interior dos quadrados foi coletado manualmente, armazenado em sacos plásticos, transportado para $\mathrm{o}$ Laboratório de Algas Marinhas da UFPB e congelado para posterior triagem. Após triado e identificado de acordo com a literatura (Joly, 1967; Pereira, 1977; Kanagawa, 1984; Nunes, 1999; Pedroza, 2004), e classificado de acordo com a 
nomenclatura atual proposta por Guiry e Guiry (2019), o material foi seco em estufa a $90^{\circ} \mathrm{C}$ por $48 \mathrm{~h}$. 0 peso seco foi medido em balança de precisão, considerando o peso mínimo de $0,01 \mathrm{~g}$, e para as espécies presentes que não atingiram esse peso mínimo, atribuiu-se o valor arbitrário de 0,005 g. A biomassa foi obtida calculando-se o peso seco por $\mathrm{m}^{2}\left(\mathrm{~kg} \mathrm{~m}^{-2}\right)$

\section{Descritores e análises estatísticas}

Foi confeccionada uma matriz de presença/ausência das espécies com base nos dados das coletas e, através dela, se determinou a riqueza específica (S), dada pela média total de espécies presentes na amostra por período e estação de coleta. A partir dos dados dessa matriz foi feita uma análise de variância (ANOVA) para avaliar variações espaço-temporais do descritor.

Os dados de biomassa total (BT), compuseram outra matriz, de onde também foram calculados os descritores de diversidade de Shannon ( $\left.\mathrm{H}^{\prime}\right)$ (Shannon, 1948) e dominância de Simpson (D) (Simpson, 1949). Foram avaliadas possíveis diferenças significativas entre estes descritores através da análise não paramétrica de Kruskal-Wallis com o teste a posteriori de Friedman (Zar, 2010), para ambos períodos. Para essa análise, foi optada pela exclusão das espécies que apresentam forte deposição de carbonato de cálcio (Halimeda opuntia, Penicilus capitatus, Amphiroa fragilissima e Tricleocarpa fragilis) da matriz, devido ao grande ruído provocado por seus altos valores de biomassa.

\section{Resultados e discussão}

\section{Sinopse dos taxa identificados}

Foi encontrado um total de 41 espécies de macroalgas: 9 Chlorophyta, 9 Ochrophyta (Phaeophyceae) e 23 Rhodophyta. 0 gênero Gracilaria foi o que apresentou maior representatividade com quatro espécies, seguido por
Caulerpa, Sargassum e Gelidium, com três espécies cada.

\section{CHLOROPHYTA}

Bryopsis pennata J. V. Lamouroux

Caulerpa cupressoides var.

lycopodium Weber-van Bosse

Caulerpa prolifera (Forsskål) J. V.

Lamouroux

Caulerpa racemosa (Forsskål) J. V.

Lamouroux

Halimeda opuntia (Linnaeus)

Lamouroux

Penicillus capitatus Lamarck

Phyllodictyon anastomosans

(Harv.) Kraft \& M. J. Winne

Udotea flabellum (J. Ellis \&

Solander) M. A. Howe

Ulva lactuca Linnaeus

\section{OCHROPHYTA}

Canistrocarpus

cervicornis

Kützing De Paula \& De Clerck

Lamouroux

Dictyopteris delicatula J. V.

Taylor

Dictyopteris jamaicensis W. R.

Kützing

Dictyota mertensii (Martius)

Lobophora variegata

(Lamouroux) Womersley ex E. C. Oliveira

Padina sanctae-crucis Børgesen

Sargassumcymosum var. nanum E.

de Paula \& E. C. Oliveira

Sargassum polyceratium

Montagne

Sargassum vulgare C. Agardh

RHODOPHYTA

J.Agardh

Alsidium seaforthii (Turner)

Trevisan

Alsidium triquetrum (S.G.Gmelin)

Amansia multifida J. V.

Lamouroux

J. V. Lamouroux

Amphiroa fragilissima (Linnaeus)

Montagne

Bostrychia radicans (Montagne)

Chondracanthus acicularis (Roth)

Fred. in Hom, Guiry, Fredericq \& Leister 
J.Agardh

Corynecladia clavata (Sonder)

Crassiphycus corneus (J.Agardh)

Gurgel, J.N.Norris \& Fredericq

J. Agardh

Cryptonemia crenulata (J. Agardh)

Agardh

Digenea simplex (Wulfen) C.

Gelidiella acerosa (Forsskål)

Feldmann \& G. Hamel

P.C. Silva

Gelidium capense (S.G. Gmelin)

Gaillon

Gelidium crinale (Hare ex Turner)

Gelidium pusillum (Stackhouse)

Le Jolis

Gracilaria cervicornis (Turner) J.

Agardh

Gracilaria domingensis (Kützing)

Sonder ex Dickie

Gracilaria sp.
Hypnea musciformis (Wulfen) J. V. Lamouroux

Kützing

Hypnea spinella (C. Agardh)

Osmundaria obtusiloba (C.

Agardh) R.E. Norris

Agardh

Scinaia furcellata (Turner) J.

Gabrielson

Solieria filiformis (Kützing) P. W.

Tricleocarpa fragilis (Linnaeus) Huisman \& R.A. Townsend

\section{Riqueza (S)}

As médias de Svariaram em maio entre 3,8 (+2,1) na estação P1 e 12,2 $(+2,57)$ na estação G1. Em outubro as médias de Svariaram entre $2,5(+1,58)$ na estação P1 e 6,3 $(+2,0)$ na estação G1. (Tabela 1).

Tabela 1. Matriz de riqueza (S) por estação e por local de coleta nos ambientes recifais de Picãozinho (P1, P2 e P3) e São Gonçalo (G1 e G2), João Pessoa/PB, nos meses de maio/2014 e outubro/2014.

\begin{tabular}{lcccccccccc}
\hline & \multicolumn{4}{c}{ Maio/2014 } & \multicolumn{4}{c}{ Outubro/2014 } \\
\cline { 2 - 11 } & P1 & P2 & P3 & G1 & G2 & P1 & P2 & P3 & G1 & G2 \\
\hline Média & 3,8 & 4,1 & 6,4 & 12,2 & 10,3 & 2,5 & 2,9 & 3,7 & 6,3 & 6,2 \\
Desvio (+) & 2,10 & 2,08 & 2,01 & 2,57 & 2,98 & 1,58 & 1,20 & 1,64 & 2,00 & 1,55 \\
\hline
\end{tabular}

A análise de variância (ANOVA) realizada com a matriz da riqueza mostrou a existência de diferenças significativas entre Picãozinho e São Gonçalo, tanto em maio ( $\mathrm{p} \leq 0,001)$ quanto em outubro $(\mathrm{p} \leq 0,01)$. As estações de coleta P1, P2 e P3 não apresentaram diferenças temporais significativas de maio para outubro $(p \geq 0,13)$. No entanto, os locais G1 e G2 de maio apresentaram diferenças significativas $(\mathrm{p} \leq 0,01)$ com G1 e G2 de outubro devido à diminuição da riqueza média nas estações de amostragem nesse mês (Figura 3).
A não existência de diferenças temporais significativas entre as estações de Picãozinho pode estar relacionada com próprio impacto ao qual essas áreas estão submetidas, pois a comunidade macrofitobêntica presente nesse ambiente já está com a riqueza comprometida. Desse modo, a queda da riqueza que naturalmente acontece durante 0 período chuvoso (Silva et al., 1987; Miranda, 2000) não afetou a comunidade de Picãozinho, dominada por poucas espécies durante todo o ano. 


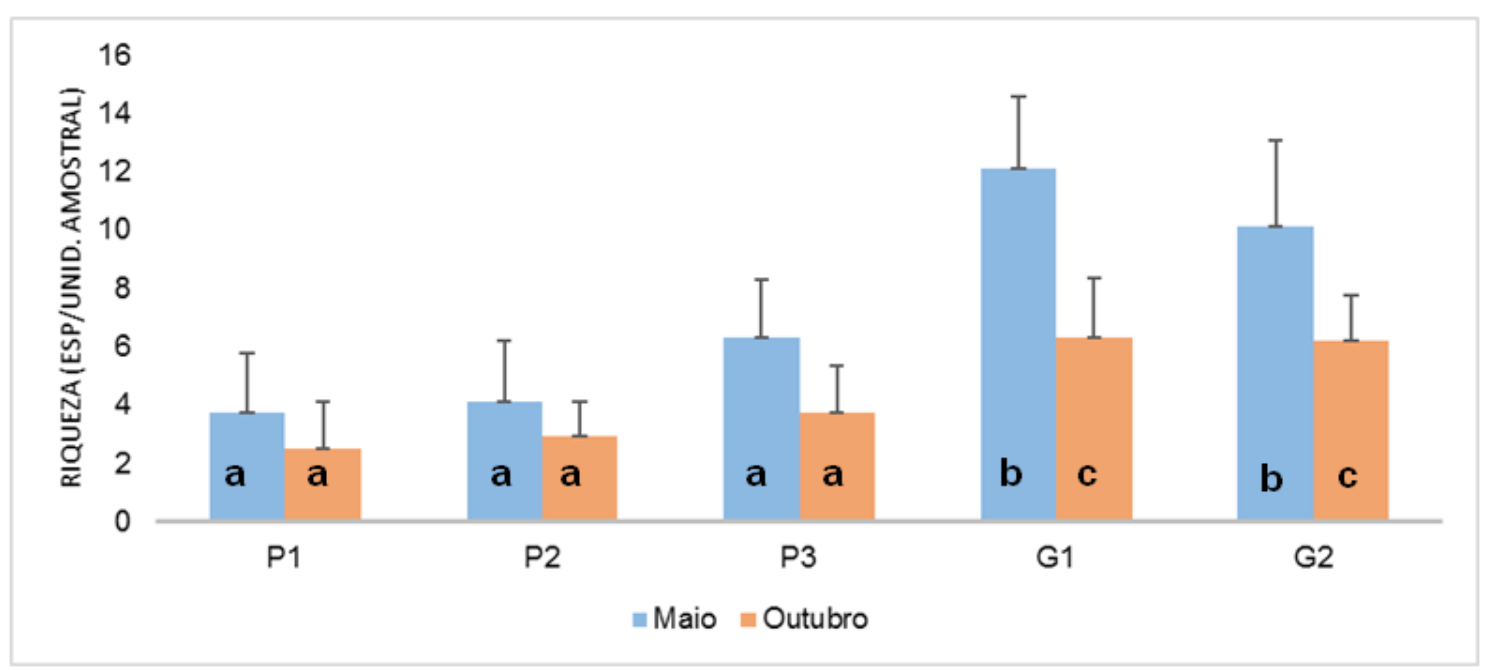

Figura 3. Média da riqueza (S) (espécies/unidade amostral) por estações de coleta/mês em Picãozinho (P1, P2 e P3) e São Gonçalo (G1 e G2). Barras indicam o desvio padrão. Letras diferentes indicam diferenças significativas (ANOVA).

Os múltiplos impactos (diretos e indiretos) do turismo em Picãozinho podem explicar a menor riqueza de espécies em relação a São Gonçalo em ambos os períodos. Dentre os impactos gerados pela atividade turística em Picãozinho estão: ancoragem sobre corais, alimentação da ictiofauna, aumento da poluição (sonora e orgânica) e da turbidez hídrica (Debeus e Crispim, 2008). A atividade de fundeio e ancoragem de embarcações (Creed e Amada Filho, 1999; Milazzo et al., 2002; Dinsdale e Harriott, 2004; Lloret et al., 2008) e o pisoteio (Brosnan e Crumrine, 1994; Keough e Quinn, 1998; Crowe et al., 2000; Milazzo et al., 2004) podem ser extremamente destrutivos para comunidades bênticas, promovendo degradação ambiental, destruição de habitats e danos ao ecossistema (Hall, 2001).

Espaços abertos deixados no substrato por danos de ancoragem podem levar longos períodos para serem recolonizados (Creed e Amado Filho, 1999). Se o dano ou distúrbio sofrido não for muito intenso, ou se o ambiente possuir grande capacidade de resiliência, é relativamente fácil para o ambiente recuperar a cobertura, mas isso pode não ocorrer quando o impacto sofrido é sistemático (Keough e Quinn, 1998), como em Picãozinho.

As estações de São Gonçalo (G1 e G2), que não apresentaram atividade turística, foram as que mostraram maior riqueza. Azevedo et al. (2011) também observaram que as áreas que apresentam atividade moderada/alta de turistas possuíam menor riqueza em comparação com áreas que não possuem essa finalidade.

0 mês de outubro apresentou queda no número de espécies em São Gonçalo em comparação com mês de maio, provavelmente devido ao período chuvoso que antecedeu a coleta, este padrão também foi percebido por Silva et al. (1987) e Miranda (2000) em estudos temporais em ambientes recifais na Paraíba. Durante o período chuvoso há diminuição das temperaturas (da água e atmosféricas), aumento da pluviosidade e nebulosidade, aumento na taxa de turbidez devido ao maior transporte de sedimentos pelos rios, dentre outras características ambientais que atuam como fatores limitantes ao desenvolvimento de diversas espécies de macroalgas (Leite e Miranda, 2018). 


\begin{tabular}{|c|c|}
\hline $\begin{array}{ccc}\text { Biomassa } & \text { Total (BT), }\end{array}$ & variaram entre $0,13 \mathrm{~kg} \mathrm{~m}^{-2}(+0,17)$ na \\
\hline Diversidade (H') e Dominância (D) & estação P1 e $0,40 \mathrm{~kg} \mathrm{~m}^{-2}(+0,17)$ na \\
\hline $\begin{array}{l}\text { As médias de BT variaram em } \\
\text { maio entre } 0,12 \mathrm{~kg} \mathrm{~m}^{-2}(+0,21) \text { na estação } \\
\text { P1 e } 0,64 \mathrm{~kg} \mathrm{~m}^{-2}(+1,07) \text { na estação } \mathrm{P} 2 \text {. }\end{array}$ & $\begin{array}{l}\text { estação G1. (Tabela 2). } 0 \text { elevado valor } \\
\text { de BT em Maio na estação P2 é devido a } \\
\text { grande ocorrência de } H \text {. opuntia. }\end{array}$ \\
\hline
\end{tabular}

Tabela 2. Matriz de Biomassa média $\left(\mathrm{kg} \mathrm{m}^{-2}\right)$ por estação e por local de coleta nos ambientes recifais de Picãozinho (P1, P2 e P3) e São Gonçalo (G1 e G2), João Pessoa/PB, nos meses de maio/2014 e outubro/2014.

\begin{tabular}{lcccccccccc}
\hline & \multicolumn{9}{c}{ Maio/2014 } & \multicolumn{1}{c}{ Outubro/2014 } \\
\cline { 2 - 12 } & P1 & P2 & P3 & G1 & G2 & P1 & P2 & P3 & G1 & G2 \\
\hline Média & 0,12 & 0,64 & 0,33 & 0,31 & 0,33 & 0,13 & 0,16 & 0,25 & 0,40 & 0,20 \\
Desvio & 0,21 & 1,07 & 0,13 & 0,16 & 0,16 & 0,17 & 0,19 & 0,08 & 0,17 & 0,15 \\
\hline
\end{tabular}

Com a exclusão das espécies calcificadas, a BT indicou diferenças significativas entre Picãozinho e São Gonçalo tanto em maio $(\mathrm{p} \leq 0,05)$ quanto em outubro $(p \leq 0,05)$. 0 teste a posteriori indicou que as estações P1 e P2 diferiram das estações G1 e G2 em maio $(p \leq 0,005)$ e em outubro $(p \leq 0,01)$, porém a estação P3 diferiu das estações P1 e P2 e não diferiu de G1 e G2 em ambos os meses ( $\mathrm{p} \leq 0,006$ para maio e $\mathrm{p} \leq 0,03$ para outubro). 0 teste ainda indicou que as estações P1 e P2 apresentaram diferenças significativas entre si em outubro $(p=0,003)$, mas não em maio $(p=0,79)$. As estações G1 e G2 não apresentaram diferenças significativas entre si, tanto em maio $(p \geq 0,43)$, quanto em outubro $(p=0,08$ ) (Figura 4).

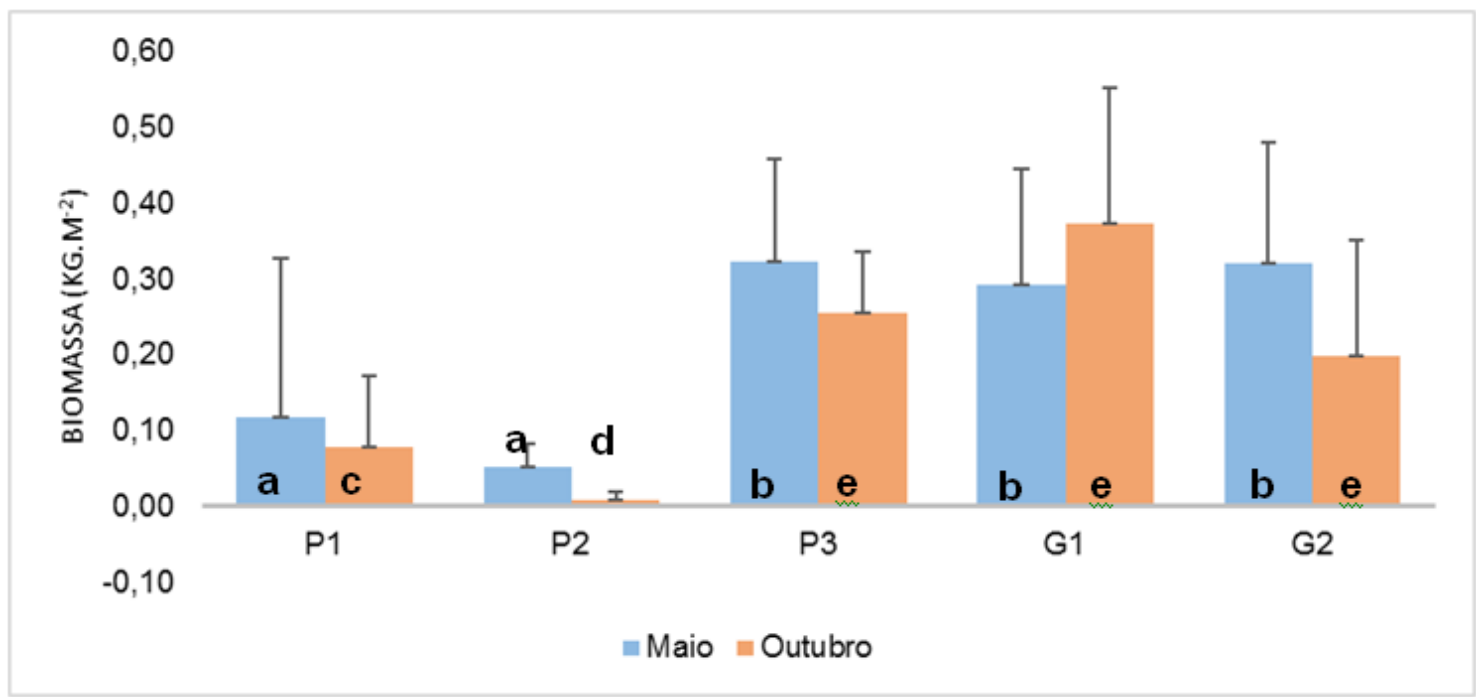

Figura 4. Biomassa média (kg.m ${ }^{-2}$ ) por estações de coleta/mês em Picãozinho (P1, P2 e P3) e São Gonçalo (G1 e G2). Barras indicam o desvio padrão. Letras diferentes indicam diferenças significativas (Kruskal-Wallis/Friedman). 
As médias de diversidade $\left(\mathrm{H}^{\prime}\right)$ variaram em maio entre $0,5(+0,49)$ na estação P1 e 1,59 (+0,34) na estação G1. Em outubro as médias de $\mathrm{H}^{\prime}$ variaram entre 0,29 (+0,38) na estação P1 e 1,01 $(+0,27)$ na estação $G 2$.

As médias de dominância (D) variaram em Maio entre 0,347 (+0,169) na estação G1 e 0,761 $(+0,238)$ na estação P1. Em outubro as médias de D variaram entre $0,484(+0,158)$ na estação G1 e 0,837 $(+0,209)$ na estação P1. Os valores detalhados de $\mathrm{H}^{\prime}$ e $\mathrm{D}$ estão na Tabela 3.

Tabela 3. Matriz de diversidade e dominância por estação e por local de coleta nos ambientes recifais de Picãozinho (P1, P2 e P3) e São Gonçalo (G1 e G2), João Pessoa/PB, nos meses de maio/2014 e outubro/2014.

\begin{tabular}{lcccccccccc}
\hline & \multicolumn{9}{c}{ Maio/2014 } & \multicolumn{6}{c}{ Outubro/2014 } \\
\cline { 2 - 12 } & P1 & P2 & P3 & G1 & G2 & P1 & P2 & P3 & G1 & G2 \\
\hline Divers. (H') & 0,50 & 0,66 & 1,08 & 1,59 & 1,31 & 0,29 & 0,34 & 0,70 & 1,00 & 1,01 \\
Desvio (H') & 0,49 & 0,50 & 0,45 & 0,38 & 0,45 & 0,39 & 0,29 & 0,41 & 0,34 & 0,27 \\
Domin. (D) & 0,761 & 0,733 & 0,454 & 0,347 & 0,406 & 0,837 & 0,786 & 0,587 & 0,484 & 0,490 \\
Desvio (D) & 0,238 & 0,284 & 0,225 & 0,169 & 0,188 & 0,209 & 0,210 & 0,241 & 0,158 & 0,145 \\
\hline
\end{tabular}

A ANOVA realizada com base na matriz de $\mathrm{H}^{\prime}$ mostrou a existência de diferenças significativas entre as estações em Picãozinho (P1 e P2) e São Gonçalo (G1 e G2) em maio/2014 $(\mathrm{p} \leq 0,016)$ e em outubro/14 ( $\mathrm{p} \leq 0,016)$. A estação P3 não apresentou diferenças significativas com todas as estações em ambos os períodos.

Temporalmente, as estações de Picãozinho (P1, P2 e P3) não apresentaram diferenças significativas entre si $(p \geq 0,52)$, assim como as estações de São Gonçalo G1 e G2 $(p \geq 0,05)$ (Figura 5).

A análise de variância (ANOVA) feita a partir da Matriz de Dominância, também mostrou a existência de diferenças espaciais significativas entre as estações de Picãozinho (P1 e P2) e São Gonçalo (G1 e G2), em maio/2014 (p s $0,04)$ e em outubro/2014 ( $\mathrm{p} \leq 0,01)$. A estação P3 novamente não apresentou diferenças significativas com as estações G1 e G2 em ambos os meses ( $p \geq 0,97$, em maio/2014; $p \geq 0,98$, em outubro/2014) apresentando diferenças significativas com as estações P1 e P2 em maio/2014 $(p=0,04)$ mas não em outubro/2014 $(p \geq 0,20)$.

Temporalmente, as estações de Picãozinho (P1, P2 e P3) não apresentaram diferenças significativas consigo mesmas ( $p \geq 0,70$ ), bem como as estações de São Gonçalo G1 e G2 (p z 0,08 ) (Figura 6). 


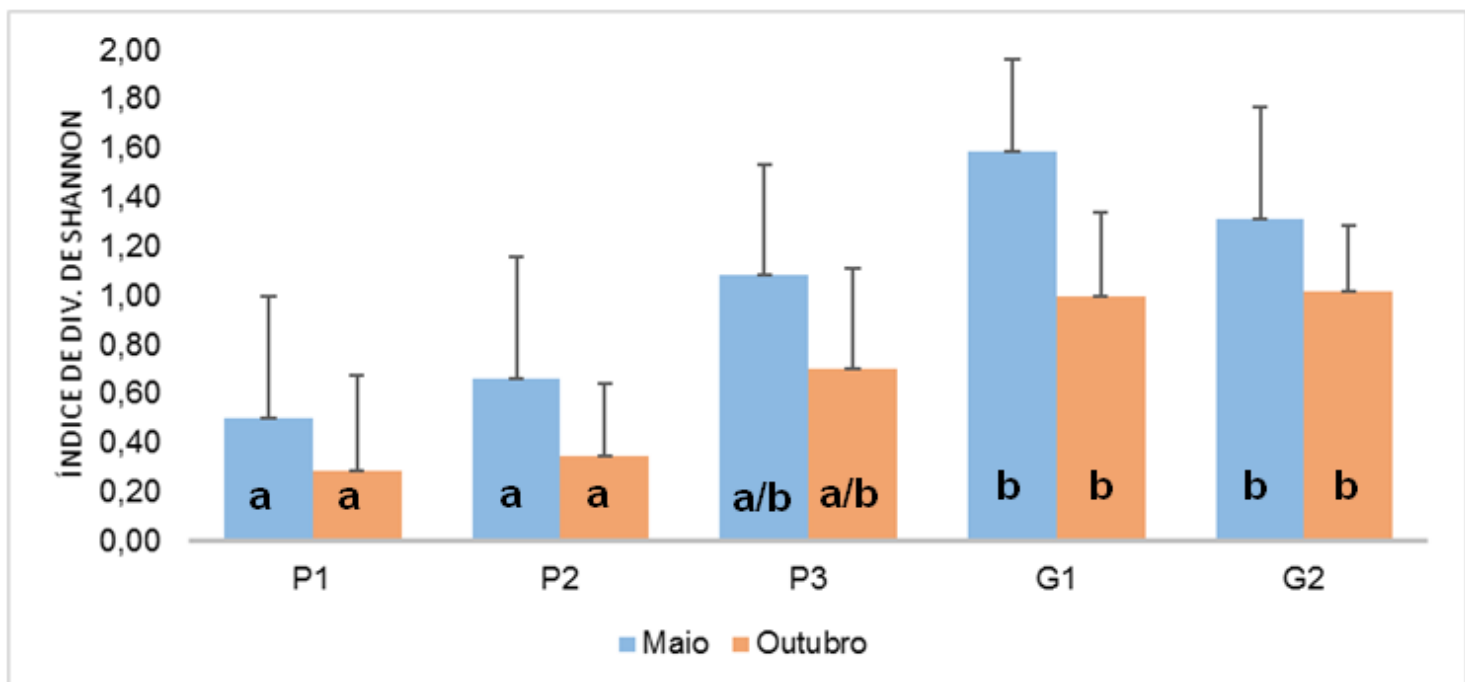

Figura 5. Diversidade (Shannon) por estações de coleta/mês em Picãozinho (P1, P2 e P3) e São Gonçalo (G1 e G2). Barras indicam o desvio padrão. Letras diferentes indicam diferenças significativas (ANOVA).

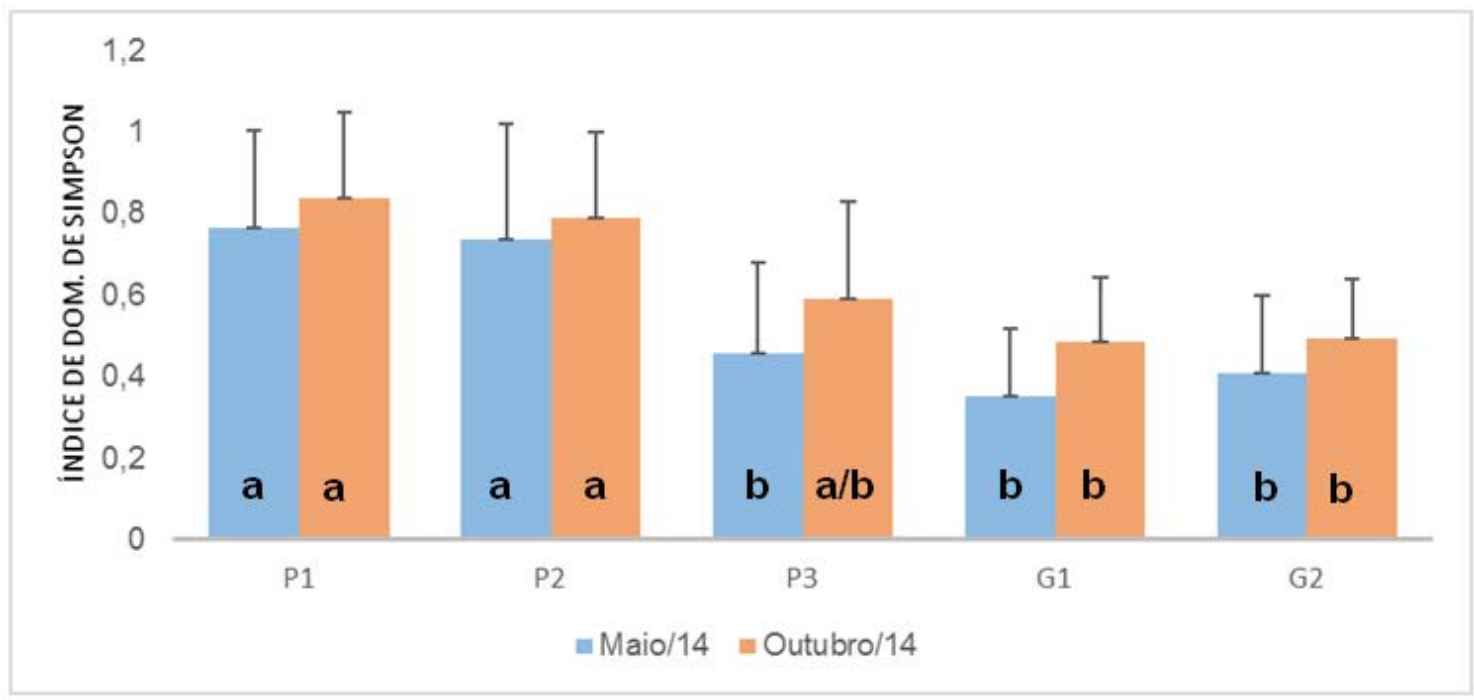

Figura 6. Dominância (Simpson) por estações de coleta/mês em Picãozinho (P1, P2 e P3) e São Gonçalo (G1 e G2). Barras indicam o desvio padrão. Letras diferentes indicam diferenças significativas (ANOVA).

Apesar de P3 compor o recife de Picãozinho, essa estação recebe pouca visita turística, apresentando uma biomassa similar as estações controle. P3 possui uma piscina natural tais quais as encontradas nas estações P1 e P2, mas para acesso faz-se necessário a passagem por cima do recife exposto, o que é desencorajado pelos guias turísticos, limitando as visitas à área a donos de barcos particulares que podem dar a volta no ambiente recifal.

P3 é mais similar as estações controle em São Gonçalo (G1 e G2), com os talos de macroalgas maiores e mais desenvolvidos, principalmente as espécies do gênero Sargassum, que segundo Borowitzka (1972), é um 
considerável indicador da não existência de impactos relevantes na área.

Como observado nas Figuras 5 e 6, a maior Diversidade é encontrada nas estações de São Gonçalo (G1 e G2), em contraste com a Dominância, que é maior nas estações com atividade turística em Picãozinho (P1 e P2). Esse padrão é esperado pois os impactos turísticos nas estações P1 e P2 limita a ocorrência de diversas espécies, provocando a dominância por um pequeno grupo de espécies resistentes aos impactos, que agrupam-se em pequenos tufos (denominados de turf), com uma baixa biomassa e na maioria das vezes composta por feofíceas, que é um grupo bastante comum em ambientes recifais (McCook, 1997; Figueiredo, 2000).

Na literatura, o turf não apresenta uma lista de espécies definitiva, ocorrendo como um grupo funcional (Hay, 1981; Airoldi, 1998; Coleman, 2002; Costa et al., 2002; Figueiredo, 2006; Figueiredo et al., 2008; Bahartan et al., 2010). Esse grupo é formado basicamente por algas filamentosas e espécimes delicadas, normalmente em estágios iniciais de desenvolvimento (Figueiredo, 2006; Figueiredo et al., 2008), que acaba constituindo a feição dominante em muitos ambientes recifais de meso e infralitoral (Adey, 1998). Essa dominância pode ser explicada pela sua resistência a invasão por outros competidores, resistência à estresses físicos e biogênicos e ainda por sua rápida recuperação da cobertura após os distúrbios, sugerindo que o turf pode dominar ambientes sob uma ampla gama de distúrbios e regimes de estresse (Airoldi, 1998).

Devido à metodologia empregada neste trabalho, não foi possível quantificar a dominância do turf nesse ambiente. Segundo Hay (1981), muitas espécies que crescem em ambientes fisicamente estressantes ou sujeitos a uma moderada herbivoria podem se desenvolver como turf, apresentando, essas mesmas espécies, comportamento não-turf quando na ausência de estressores, o que dificulta a comparação desse grupo funcional através do descritor biomassa.

Considerando o elevado potencial turístico, o recife de Picãozinho é particularmente vulnerável a influências antropogênicas, como por exemplo o pisoteio dos organismos bentônicos por centenas de turistas que visitam o local todo o ano. Para Brosnan e Crumrine (1994) e Crowe et al. (2000), há uma relação direta entre esse tipo de impacto e os danos nos habitats. Com o pisoteio, a quantidade de cracas, mexilhões e algas diminuem consideravelmente, além de causar o branqueamento de corais, ocasionando um aumento de espaços vazios e a consequente colonização por espécies oportunistas (Crowe et al., 2000), tal como o turf, que é mais resistente (Brosnan e Crumrine, 1994). Milazzo et al. (2007), Casu et al. (2006)e Davenport e Davenport(2006) observaram que áreas impactadas pelo pisoteio possuem biomassa e tamanho do talo das macroalgas menores em comparação com áreas não pisoteadas.

Desde 28 de dezembro de 2018, os recifes de Picãozinho e São Gonçalo compõem a nova UC marinha da Paraíba, a APA Naufrágio Queimado (Decreto $\mathrm{n}$ 38.931/2018), passando a ser administrados pela SUDEMA em articulação com os órgãos federais, estaduais e municipais, bem como organizações não governamentais (Paraíba, 2018). Para os recifes, a criação da UC é importante para proteção da diversidade recifal, sustentabilidade no uso dos recursos naturais e ordenamento do turismo e dos demais usos do recife (Leite, 2019), porém destaca-se que durante a realização do presente trabalho, os recifes de Picãozinho e de São Gonçalo ainda não estavam legalmente protegidos pela UC, sendo avaliados como áreas sob domínio da União com base na Lei do Mar Territorial, a Lei no 8.617/1993 (Brasil, 1993). Este panorama torna possível comparações entre as variações espaço-temporais da comunidade macrofitobentônica antes e 
após a criação da UC, servindo de base para o (re)planejamento de ações que objetivem à melhoria da gestão visando $o$ desenvolvimento socioeconômico e ambiental dos recifes costeiros avaliados. Articulações efetivas entre os entes federados para planejamento e, principalmente, implementação de ações de gerenciamento costeiro, fazem-se necessárias, visto à complexidade demandada para a gestão de ambientes recifais. Diante dos múltiplos usos aos quais os recifes avaliados estão submetidos, se faz necessário o entendimento de como a gestão influencia e é influenciada pelo estado de conservação ambiental.

\section{Conclusões}

A comparação entre os ambientes recifais de Picãozinho e São Gonçalo evidencia que a comunidade macrofitobêntica é sensível aos impactos no ambiente em que está inserida. De origem natural ou antropogênica, os impactos influenciam na distribuição e desenvolvimento das macroalgas, alterando padrões na estrutura das comunidades.

0 ambiente recifal de Picãozinho sofre com as mudanças resultantes da atividade turística no local, ocasionando níveis de riqueza, biomassa e diversidade de espécies menores em comparação com áreas controle para a atividade turística. Além disso, as estações impactadas pelo turismo são dominadas por poucas espécies resistentes aos impactos, com predomínio da fitofisionomia de turf.

A utilização de macroalgas como bioindicadoras de qualidade ambiental é válida por serem sensíveisaos impactos do ambiente em que estão inseridas, fornecendo um panorama atual do estado de conservação local. Estimula-se a adoção de comunidade de macroalgas no monitoramento dos impactos da nova APA Naufrágio Queimado, podendo incorporar o método da bioindicação ao futuro plano de manejo da UC.

\section{Conflito de interesses}

Os autores declaram não haver conflito de interesses.

\section{Referências}

Adey, W. H. Coral reefs: Algal structured and mediated ecosystems in shallow, turbulent, alkaline waters. Journal of Phycology, v. 34, p. 393-406, $1998 . \quad$ https://doi.org/ 10.1046/j.1529-8817.1998.340393.x

Airoldi, L. Roles of disturbance, sediment stress, and substratum retention on spatial dominance in algal turf. Ecology, v. 79, n. 8, p. 2759-2770, 1998 . https://doi.org/ 10.1890/0012-9658(1998)079[2759: RODSSA]2.0.CO;2

Akcali, I.; Kucuksezgin, F. A biomonitoring study: Heavy metals in macroalgae from Eastern Aegean Coastal Areas. Marine Pollution Bulletin, v. 62, n. 3, p. 637-645, 2011. https://doi.org/10.1016/j.marpolbul. 2010.12.021

Ar Gall, E.; Le Duff, M.; Sauriau, P. G.; Casamajor, M. N.; Gevaert, F.; Poisson, E.; Hacquebart, P.; Joncourt, Y.; Barillé, A. L.; Buchet, R.; Bréret, M.; Miossec, L. Implementation of a new index to assess intertidal seaweed communities as bioindicators for the European Water Framework Directory. Ecological Indicators, v. 60, p. 162-173, 2016. https://doi.org/10.1016/j.ecolind.2015.06.0 35

Areces Mallea, A. J.; Cocentino, A. L. M.; Reis, T. N. V.; Vasconcelos, E. R. T. P. P; GuimarãesBarros, N. C.; Fujii, M. T. Las macroalgas como bioindicadoras da calidad ambiental y cambios climáticos. Guia prática. Brazilian Journal of Ecology, v. Especial, p. 1-64, 2015.

Azevedo, C. A. A.; Carneiro, M. A. A.; Oliveira, S. R.; Marinho-Soriano, E. Macrolgae as an indicator of the environmental health of the Pirangi reefs, Rio Grande do Norte, Brazil. Revista Brasileira Farmacognosia, v. 21, n. 2, p. 323-328, 2011. https://doi.org/ 10.1590/S0102-695X2011005000071

Bahartan, K.; Zibdah, M.; Ahmed, Y.; Israel, A.; Brickner, I.; Abelson, A. Macroalgae in the coral reefs of Eilat (Gulf of Aqaba, Red Sea) as a possible indicator of reef degradation. Marine Pollution Bulletin, v. 60 , n. 5, 
p. 759-764, 2010. https://doi.org/10.1016/ j.marpolbul.2009.11.017

Borowitzka, M. A. Intertidal algal species diversity and the effects of pollution. Australian Journal of Marine and Freshwater Research, v. 23, n. 2, p. 73-84, 1972. https://doi.org/10.1071/MF9720073

Brasil. Lei no 8.617, de 4 de janeiro de 1993. Dispõe sobre o mar territorial, a zona contígua, a zona econômica exclusiva e a plataforma continental brasileiros, e dá outras providências. Disponível em: <http://www.planalto.gov.br/ccivil_03/LEIS /L8617.htm>. Acesso em: 01 fev. 2019.

Brosnan, D. M.; Crumrine, L. L. Effects of human trampling on marine rocky shore communities. Journal of Experimental Marine Biology and Ecology, v. 177, n. 1, p. 79-97, 1994. https://doi.org/10.1016/00220981(94)90145-7

Castro, C. B.; Zilberberg, C. Recifes brasileiros, sua importância e conservação. In: Zilberberg, C.; Abrantes, D. P.; Machado, L. F.; Marangoni, L. F. B.; Marques, J. A. (Eds.). Conhecendo os recifes brasileiros: Rede de Pesquisas Coral Vivo. Rio de Janeiro: UFRJ, Museu Nacional, 2016. (Série Livros Museu Nacional). p. 17-26.

Casu, D.; Ceccherelli, G.; Curini-Galletti, M.; Castelli, A. Human exclusion from rocky shores in a mediterranean marine protected area (MPA): An opportunity to investigate the effects of trampling. Marine Environmental Research, v. 62, p. 15-32, 2006.

Coleman, M. A. Small-scale spatial variability in intertidal and subtidal turfing algal assemblages and the temporal generality of these patterns. Journal of Experimental Marine Biology and Ecology, v. 267, p. 5374, 2002. https://doi.org/10.1016/S00220981(01)00358-6

Costa, C. F.; Sassi, R.; Costa, M. A. J.; Brito, A. C. L. Recifes costeiros da Paraíba, Brasil: usos, impactos e necessidades de manejo no contexto da sustentabilidade. Gaia Scientia, v. 1, n. 1, p. 37-45, 2007.

Costa, O. S.; Attrill, M. J.; A. G. Pedrini, A. G.; De-Paula, J. C. Spatial and Seasonal Distribution of Seaweeds on Coral Reefs from Southern Bahia, Brazil. Botanica Marina, v. 45 , p. $346-355,2002$.
Creed, J. C.; Amado Filho, G. M. Disturbance and recovery of the macroflora of a seagrass (Halodule wrightii Ascherson) meadow in the Abrolhos Marine National Park, Brazil: An experimental evaluation of anchor damage. Journal of Experimental Marine Biology and Ecology, v. 235, p. 285-306, 1999. https://doi.org/10.1016/S0022-0981(98) 00188-9

Crowe, T.P.; Thompson, R. C.; Bray, S.; Hawkins, S. J. Impacts of anthropogenic stress on rocky intertidal communities. Journal of Aquatic Ecosystem Stress and Recovery, v. 7, n. 4, p. 273-297, 2000. https://doi.org/ 10.1023/A:1009911928100

Davenport, J.; Davenport, J. The impact of tourism and personal leisure transport on coastal environments: A review. Estuarine, Coastal and Shelf Science, v. 67, n. 1/2, p. 280-292, 2006. https://doi.org/10.1016/ j.ecss.2005.11.026

Debeus, G. Turismo sustentável como alternativa de desenvolvimento e conservação do meio ambiente em Picãozinho - Município de João Pessoa. João Pessoa: Universidade Federal da Paraíba, 2008. (Dissertação de mestrado).

Debeus, G.; Crispim, M. C. 0 turismo nas piscinas naturais de Picãozinho, João Pessoa, PB: percepções conflitos e alternativas. Revista de Estudos Ambientais, v. 10, n. 1, p. 21-32, 2008. https://doi.org/10.7867/ 1983-1501.2008v10n1p21-32

Dinsdale, E. A.; Harriott, V. J. Assessing anchor damage on coral reefs: A case study in selection of environmental indicators. Environmental Management, v. 33, n. 1, p. 126-139, 2004. https://doi.org/10.1007/ s00267-003-3056-9

Dokulil, M. T. Algae as ecological bio-indicators. In: Markert, B. A.; Breure, A. M.; Zechmeinster, H. G. (Eds.). Bioindicators and biomonitors. New York: Elsevier Science, 2003.

Ferreira, B. P.; Maida, M. Monitoramento dos recifes de coral do Brasil: situação atual e perspectivas. Brasília: MMA/SBF, 2006.

Figueiredo, M. A. O. Recifes de corais ou recifes de algas? Ciências Hoje, v. 166, n. 28, p. $74-75,2000$. 
Figueiredo, M. A. O. Diversity of macrophytes in the Abrolhos bank, Brazil. In: Dutra, G. F.; Allen, G. R.; Werner, T.; McKenna, S. A. (Eds.). $A$ rapid marine biodiversity assessment of the Abrolhos Bank, Bahia, Brazil. Washington, DC: Conservation International, 2006. (RAP Bulletin of Biological Assessment, 38). p.67-74.

Figueiredo, M. A. O.; Horta, P. A.; Pedrini, A. G.; Nunes, J. M. C. Benthic marine algae of the coral reefs of Brazil: A literature review. Oecologia Australis, v. 12, n. 2, p. 258-269, 2008.

Graham, N. A. J.; Wilson, S. K.; Jennings, S.; Polunin, N. V. C.; Bijoux, J. P.; Robinson, J. Dynamic fragility of oceanic coral reef ecosystems. PNAS, Proceedings of the National Academy of Sciences, v. 103, n. 22, p. 8425-8429, 2006. https://doi.org/ $10.1073 /$ pnas.0600693103

Guiry, M. D.; Guiry, G. M. Algae Base. Worldwide electronic publication, National University of Ireland, Galway. 2019. Disponível em: <http://www.algaebase.org>. Acesso em: 12 fev. 2019.

Hall, C. M. Trends in ocean and coastal tourism: The end of the last frontier? Ocean \& Coastal Management, v. 44, n. 9/10, p. 601-618, 2001. https://doi.org/10.1016/ S0964-5691(01)00071-0

Hay, M. E. The functional morphology of turfforming seaweeds: Persistence in stressful marine habitats. Ecology, v. 62, n. 3, p. 739750, 1981. https://doi.org/10.2307/ 1937742

Jennings, S. Patterns and prediction of population recovery in marine reserves. Reviews in Fish Biology and Fisheries, v. 10 , p. 209-231, 2000. https://doi.org/ 10.1023/A:1016619102955

Joly, A. B. Gênero de algas marinhas da Costa Atlântica Latino-Americana. São Paulo: USP, 1967.

Kanagawa, A. I. Clorofíceas marinhas bentônicas do Estado da Paraíba - Brasil. São Paulo: Universidade de São Paulo, Instituto de Biociências, 1984. (Tese de doutorado).
Keough, M. J.; Quinn, G. P. Effects of periodic disturbances from trampling on rocky intertidal algal beds. Ecological Applications, v. 8, n. 1, p. 141-161, 1998. https://doi.org/10.2307/2641317

Leão, Z. M. A. N.; Kikuchi, R. K. P.; Testa, V. Corals and coral reefs of Brazil. In: Cortés, J. (Ed.). Latin American coral reefs. Amsterdam: Elsevier, 2003. p. 9-52. https://doi.org/10.1016/B978-0444513885/50003-5

Leite, D. S. L. Estado de conservação dos recifes costeiros da Paraíba, Brasil: a relação com a política e a gestão ambiental. João Pessoa: Programa de Pós-Graduação em Desenvolvimento e Meio Ambiente, Universidade Federal da Paraíba, 2019. (Dissertação de mestrado).

Leite, D. S. L.; Miranda, G. E. C. Avaliação e proposta de monitoramento do estado de conservação de ambiente recifal costeiro do Estado da Paraíba, Brasil: contribuições para gestão ambiental. Revista Brasileira de Gestão Ambiental e Sustentabilidade, v. 5, n. 11, p.949-967, 2018. https://doi.org/ 10.21438/rbgas.051112

Lloret, J.; Zaragoza, N.; Caballero, D.; Riera, V. Impacts of recreational boating on the marine environment of Cap de Creus (Mediterranean Sea). Ocean \& Coastal Management, v. 51, n. 11, p. 749-754, 2008. https://doi.org/10.1016/j.ocecoaman.2008.0 7.001

Lourenço, L. J. S. Proposta de zoneamento e capacidade de carga para o Parque Estadual Marinho de Areia Vermelha. João Pessoa: Programa de Pós-Graduação em Desenvolvimento e Meio Ambiente, Universidade Federal da Paraíba, João Pessoa, 2010. (Dissertação de mestrado).

McClanahan, T. R. Bleaching damage and recovery potential of Maldivian coral reefs. Marine Pollution Bulletin, v. 40, n. 7, p. 587597, 2000. https://doi.org/10.1016/S0025326X(00)00064-3

McCook, L. J. Macroalgae, nutrients and phase shifts on coral reefs: Scientific issues and management consequences for the Great Barrier Reef. Coral Reefs, v. 18, p. 157-367, 1997. https://doi.org/10.1007/s0033800 50213 
McManus, J. W.; Polsenberg, J. F. Coral-algal phase shifts on coral reefs: Ecological and environmental aspects. Progress in Oceanography, v. 60, n. 2/4, p. 263-279, 2004. https://doi.org/10.1016/j.pocean. 2004.02.014

Milazzo, M.; Badalamenti, F.; Riggio, S.; Chemello, R. Patterns of algal recovery and small-scale effects of canopy removal as a result of human trampling on a Mediterranean rocky shallow community. Biological Conservation, v. 117, n. 2, p. 191202, 2004. https://doi.org/10.1016/S00063207(03)00292-1

Milazzo, M.; Chemello, R.; Badalamenti, F.; Camarda, R.; Riggio, S. The impact of human recreational activities in marine protect areas: What lesson should be learnt in the Mediterranean Sea? Marine Ecology, v. 23, Suppl. 1, p. 280-290, 2002.

Miranda, G. E. C. Avaliação do impacto da exploração (simulada) da alga agarófita Gracilaria caudata J. Agardh (Rhodophyta) no litoral do Estado da Paraíba. São Paulo: Universidade de São Paulo, Instituto de Biociências, 2000. (Dissertação de mestrado).

Nunes, J. M. C. Phaeophyta da Região Metropolitana de Salvador, Bahia, Brasil. São Paulo: Universidade de São Paulo, Instituto de Biociências, 1999. (Tese de doutorado).

Ortega, J. L. G. Algas. In: Espino, G. L.; Pulido, S. H.; Pérez, J. L. C. (Eds.). Organismos indicadores de la calidad del agua y de la contaminación (bioindicadores). Playa y Valdés, Mexico: UNAM, 2000. p. 109-193.

Paraíba. Decreto no 38.931, de 28 de dezembro de 2018. Cria a Área de Proteção Ambiental Naufrágio Queimado, e dá outras providências. Disponível em: <http://static. paraiba.pb.gov.br/2019/01/Diario-Oficial30-12-2018-Total.pdf\#page=16>. Acesso em: 01 fev. 2019.

Pedroza, K. K. A. L. Levantamento florístico das feofíceas no litoral do Estado da Paraíba - Brasil. Recife: Universidade Federal Rural de Pernambuco, 2004. (Dissertação de mestrado).

Pereira, S. M. B. Rodofíceas marinhas da Ilha de Itamaracá e arredores (Estado de Pernambuco - Brasil). São Paulo:
Universidade de São Paulo, Instituto de Biociências, 1977. (Tese de doutorado).

Pinedo, S.; García, M.; Satta, M. P.; De Torres, M.; Ballesteros, E. Rocky-shore communities as indicators of water quality: A case study in the Northwestern Mediterranean. Marine Pollucion Bulletin, v. 55, n. 1/6, p. 126-135, 2007. https://doi.org/10.1016/j.marpolbul. 2006.08.044

Shannon, C.E. A mathematical theory of communication. Bell System Technical Journal, v. 27, n. 3, p. 379-423, 623-656, 1948. https://doi.org/10.1002/j.15387305.1948.tb01338.x

Silva, M. B. Dinâmica dos padrões sucessionais do macrofitobentos em áreas marinhas sob diferentes regimes de proteção. João Pessoa: Universidade Federal da Paraíba, Programa de Pós-Graduação em Ecologia e Monitoramento Ambiental, 2013. (Dissertação de mestrado).

Silva, R. L.; Pereira, S. M. B.; Oliveira Filho, E. C.; Eston, V. R. Structure of a commercial bed of Gracilaria spp in Northeastern Brazil. Botanica Marina, v. 30, n. 6, p. 517-523, 1987.

Simpson, E. H. Measurement of diversity. $\begin{array}{llll}\text { Nature, } & \text { v. } 163, \quad \text { p. 688, } & 1949 .\end{array}$ https://doi.org/10.1038/163688a0

Souza, A. T.; Ilarri, M. I.; Medeiros, P. M.; Grempel, R. G.; Rosa, R. S; Sampaio, C. L. S. Fishes (Elasmobranchii and Actinopterygii) of Picãozinho reef, Northeastern Brazil, with notes on their conservation status. Zootaxa, v. 1608 , p. 11-19, 2007. https://doi.org/ 10.11646/zootaxa.1608.1.2

Stevenson, R. J.; Smol, R. P. Use of algae in environmental assessments. In: Wehr, J. D.; Sheath, R. G. (Eds.). Freshwater algae of North America. New York: Elsevier Science, 2003. p. $775-804$.

Vanclay, J. Indicator groups and faunal richness. Forest Biometry, Modelling and Information Sciences, v. 1, p. 105-113, 2004.

Vasconcelos, E. R. T. P. P.; Vasconcelos, J. B.; Reis, T. N.; Cocentino, A. L. M.; Areces Mallea, A. J.; Martins, G. M.; Isabel Neto, A.; Fujii, M. T. Macroalgal responses to coastal urbanization: Relative abundance of indicator species. Journal of Applied Phycology, v. 9, n. 2, p. 893-903, 2019. 
https://doi.org/10.1007/s10811-018-1639-

3

Wells, E.; Wood, P.; Wilkinson, M.; Scanlan, C. The use of macroalgal species richness and composition on intertidal rocky seashores in the assessment of ecological quality under the European Water Framework Directive. Marine Pollution Bulletin, v. 55, p. 151-161, 2007. https://doi.org/10.1016/j.marpolbul. 2006.08.031

Zar, J. H. Biostatistical analysis. 5. ed. New York: Prentice Hall, 2010. 
Informação da Licença: Este é um artigo Open Access distribuído sob os termos da Licença Creative Commons
(CC) Attribution, que permite uso irrestrito, distribuição e reprodução em qualquer meio, desde que a obra original seja devidamente citada. 